International Mathematical Forum, Vol. 8, 2013, no. 27, 1345 - 1351

HIKARI Ltd, www.m-hikari.com

http://dx.doi.org/10.12988/imf.2013.35109

\title{
Concircular Trasformation of a Quarter Symmetric Metric Connection in an SP-Sasakian Manifold
}

\author{
Kalpana and Priti Srivastava \\ Department of Mathematics \\ Banaras Hindu University, Varanasi-221 005, India \\ kalp_2505@yahoo.in, priti143me@yahoo.co.in
}

Copyright (C) 2013 Kalpana and Priti Srivastava. This is an open access article distributed under the Creative Commons Attribution License, which permits unrestricted use, distribution, and reproduction in any medium, provided the original work is properly cited.

\begin{abstract}
In 1982, P. Stavre [8] defined semi-symmetric S-concircular and S-conharmonic connections and established some results. Anologous to this we have defined a concircular transformation on quarter symmetric metric connection in an SP-Sasakian manifold. The invariance property of the $\eta$ Einstein tensor and conformal curvature tensor has been shown.
\end{abstract}

Mathematics Subject Classification: 53C07

Keywords: Conformal curvature tensor, $\eta$-Einstein, P-Sasakian manifold, Quarter symmetric metric connection, SP-Sasakian manifold

\section{Preliminaries}

An n-dimensional differentiable manifold $M$ is called an almost para-contact manifold if it admits an almost para-contact structure $(\phi, \xi, \eta)$ consisting of a $(1,1)$ tensor field $\phi$, a vector field $\xi$ and 1-form $\eta$ satisfying

$$
\begin{gathered}
\phi^{2} X=X-\eta(X) \xi ; \quad \phi \circ \xi=0 ; \\
\eta(\xi)=1 ; \quad \eta \circ \phi=0 ; \quad \operatorname{rank}(\phi)=n-1
\end{gathered}
$$

for all $X \in T M$

Let $g$ be the Riemannian metric satisfying 


$$
g(\phi X, \phi Y)=g(X, Y)-\eta(X) \eta(Y)
$$

or equivalently

$$
g(\phi X, Y)=g(X, \phi Y) ; \quad g(X, \xi)=\eta(X)
$$

for all $\mathrm{X}, \mathrm{Y} \in \mathrm{TM}$. Then $\mathrm{M}$ becomes an almost para-contact Riemannian manifold equipped with the almost para-contact Riemannian structure $(\phi, \xi, \eta, g)$ $[7]$.

If we define,

$$
{ }^{\prime} \phi(X, Y)=g(\phi X, Y)
$$

then in addition to the above equations, we have

$$
{ }^{\prime} \phi(X, Y)={ }^{\prime} \phi(Y, X) ; \quad{ }^{\prime} \phi(\phi X, \phi Y)={ }^{\prime} \phi(X, Y)
$$

An almost para-contact Riemmenian manifold is called a P-Sasakian manifold [1] if it satisfies

$$
\left(\nabla_{X} \phi\right) Y=-g(X, Y) \xi-\eta(Y) X+2 \eta(Y) \eta(X) \xi \quad \forall X, Y \in T M
$$

where $\nabla$ is a Levi-civita connection of the Riemannian metric.From the above equation it follows that

$$
\begin{gathered}
\nabla_{X} \xi=\phi X \\
\left(\nabla_{X} \eta\right) Y=g(X, \phi Y)=\left(\nabla_{Y} \eta\right) X
\end{gathered}
$$

Especially, a P-Sasakian manifold $M$ is called a special para-Sasakian manifold or briefly an SP-Sasakian manifold [6] if $M$ admits a 1-form $\eta$ satisfying

$$
\left(\nabla_{X} \eta\right) Y=-g(X, Y)+\eta(X) \eta(Y)
$$

For SP-Sasakian manifold we have [2]

$$
{ }^{\prime} \phi(X, Y)=-g(X, Y)+\eta(X) \eta(Y)
$$

In an n-dimensional P-Sasakian manifold $M$, the curvature tensor $R$, the Ricci tensor $S$ satisfy (see [1], [2] and [9])

$$
\eta(R(X, Y) Z)=g(X, Z) \eta(Y)-g(Y, Z) \eta(X)
$$




$$
\begin{gathered}
S(X, \xi)=-(n-1) \eta(X) \\
R \xi=-(n-1) \xi
\end{gathered}
$$

for any vector fields $X, Y, Z \in T M$.

A linear connection $\tilde{\nabla}$ in a Riemannian manifold is said to be quarter symmetric connection [5] if its torsion tensor $T$ satisfies

$$
T(X, Y)=\tilde{\nabla}_{X} Y-\tilde{\nabla}_{Y} X-[X, Y]=p(Y) t X-p(X) t Y
$$

where $\mathrm{p}$ is 1 -form and $\mathrm{t}$ is a tensor of type $(1,1)$. A linear connection $\tilde{\nabla}$ is called a metric connection [10] iff

$$
\tilde{\nabla}_{X} g=0
$$

Definition 1.1. A linear connection $\tilde{\nabla}$ satisfying (14) and (15) is called a quarter symmetric metric connection [4].

Let $\nabla$ be the Riemannian connection with respect to $g$, then for quarter symmetric metric connection, we have

$$
\tilde{\nabla}_{X} Y=\nabla_{X} Y+p(Y) t X-{ }^{\prime} T(X, Y) P
$$

where $\quad ' T(X, Y)=g(t(X), Y) \quad$ and $\quad p(X)=g(X, P)$

Putting $t(X)=\phi(X)$, we get

$$
\tilde{\nabla}_{X} Y=\nabla_{X} Y+p(Y) \phi X-{ }^{\prime} \phi(X, Y) P
$$

\section{Curvature Tensor}

Let $R$ and $\tilde{R}$ be the curvature tensors of the connections $\nabla$ and $\tilde{\nabla}$ respectively, then we have

$$
\begin{aligned}
& R(X, Y, Z)=\nabla_{X} \nabla_{Y} Z-\nabla_{Y} \nabla_{X} Z-\nabla_{[X, Y]} Z \\
& \tilde{R}(X, Y, Z)=\tilde{\nabla}_{X} \tilde{\nabla}_{Y} Z-\tilde{\nabla}_{Y} \tilde{\nabla}_{X} Z-\tilde{\nabla}_{[X, Y]} Z
\end{aligned}
$$


Using (4), (10), (16), (18) and (19), we immediately get the following proposition.

Proposition 2.1. For the quarter symmetric connection (17) the curvature tensor $\tilde{R}$ of $\tilde{\nabla}$ is given by

$$
\begin{aligned}
\tilde{R}(X, Y, Z)=R(X, Y, Z)+Q(X, Z) \phi Y-Q(Y, Z) \phi X \\
\\
-{ }^{\prime} \phi(Y, Z) Q X+{ }^{\prime} \phi(X, Z) Q Y
\end{aligned}
$$

where,

$$
Q(X, Y)=g(Q X, Y)=\left(\nabla_{X} p\right) Y-p(Y) p(\phi X)+\frac{1}{2}{ }^{\prime} \phi(X, Y) p(P)-p(Y) \eta(X)
$$

Definition 2.1. Let the quarter symmetric metric connection $\tilde{\nabla}$ satisfies

$$
Q(X, Y)=f g(X, Y)
$$

where $\mathrm{f}$ is a scalar function, then $\tilde{\nabla}$ is called quarter symmetric metric connection with concircular transformation.

Theorem 2.1. If a quarter symmetric metric connection, in an SP-Sasakian manifold satisfies (21), then the curvature tensors are related by

$$
\tilde{R}(X, Y, Z)-\tilde{L}(X, Y, Z)=R(X, Y, Z)-L(X, Y, Z)
$$

where

$$
\begin{aligned}
L(X, Y, Z)=\frac{r}{2(n-1)^{2}}[g(X, Z) \phi Y- & g(Y, Z) \phi X \\
& \left.-{ }^{\prime} \phi(Y, Z) X+{ }^{\prime} \phi(X, Z) Y\right]
\end{aligned}
$$

and $\mathrm{r}, \tilde{r}$ be the scalar curvatures of the connections $\nabla$ and $\tilde{\nabla}$ respectively.

Proof. Let us consoder a transformation (17) satisfying (21). Then (20) gives

$$
\begin{aligned}
\tilde{R}(X, Y, Z)=R(X, Y, Z+f[g(X, Z) \phi Y & -g(Y, Z) \phi X \\
& \left.-{ }^{\prime} \phi(Y, Z) X+{ }^{\prime} \phi(X, Z) Y\right]
\end{aligned}
$$


Contracting (24), we get

$$
\begin{aligned}
\tilde{\operatorname{Ric}}(Y, Z)=\operatorname{Ric}(Y, Z)+f[g(\phi Y, Z)+(n-1) g(Y, Z) & \\
& \left.-n^{\prime} \phi(Y, Z)+{ }^{\prime} \phi(Y, Z)\right]
\end{aligned}
$$

From (4) and (25), we get

$$
\tilde{\operatorname{Ric}}(Y, Z)=\operatorname{Ric}(Y, Z)+f\left[(n-1) g(Y, Z)-(n-2)^{\prime} \phi(Y, Z)\right]
$$

This gives

$$
\tilde{R} Y=R Y+f[(n-1) Y-(n-2) \phi Y]
$$

Again by the contraction of (27), we get

$$
f=\frac{\tilde{r}-r}{2(n-1)^{2}}
$$

From (23), (25) and (28), we get (22).

Theorem 2.2. If $\tilde{\nabla}$ is a quarter symmetrc metric connection in an SP-Sasakian manifold satisfying (21), then the conformal curvature tensor is invariant of the transformation (17).

Proof. From (24), (26) and (27), we have

$$
\begin{aligned}
\tilde{R}(X, Y, Z)= & R(X, Y, Z)+g(X, Z)\left(\frac{\tilde{R} Y-R Y-f(n-1) Y}{-(n-2)}\right) \\
& -g(Y, Z)\left(\frac{\tilde{R} X-R X-f(n-1) X}{-(n-2)}\right) \\
& -\left(\frac{\tilde{\operatorname{Ric}}(Y, Z)-\operatorname{Ric}(Y, Z)-f(n-1) g(Y, Z)}{-(n-2)}\right) X \\
& +\left(\frac{\tilde{\operatorname{Ric}}(X, Z)-\operatorname{Ric}(X, Z)-f(n-1) g(X, Z)}{-(n-2)}\right) Y
\end{aligned}
$$

From (28) and (29), we get 


$$
\begin{array}{r}
\tilde{R}(X, Y, Z)+\frac{1}{(n-2)}[g(X, Z) \tilde{R} Y-g(Y, Z) \tilde{R} X-\tilde{\operatorname{Ric}}(Y, Z) X \\
+\tilde{\operatorname{Ric}}(X, Z) Y]-\frac{\tilde{r}}{(n-1)(n-2)}[g(X, Z) Y-g(Y, Z) X] \\
=R(X, Y, Z)+\frac{1}{(n-2)}[g(X, Z) R Y-g(Y, Z) R X-\operatorname{Ric}(Y, Z) X \\
+\operatorname{Ric}(X, Z) Y]-\frac{r}{(n-1)(n-2)}[g(X, Z) Y-g(Y, Z) X]
\end{array}
$$

Which shows the invariance of conformal curvature tensor.

Definition 2.2. An almost para-contact metric manifold is said to be $\eta$ Einstein if the Ricci tensor $S$ satisfies,

$$
S(X, Y)=a g(X, Y)+b \eta(X) \eta(Y)
$$

where $\mathrm{a}$ and $\mathrm{b}$ are smooth functions on the manifold $\mathrm{M}[6]$.

Theorem 2.3. If $\tilde{\nabla}$ is a quarter symmetric metric connection in an SPSasakian manifold satisfying (21), then the tensor of $\eta$-Einstein given by (31) is invariant of the transformation (17).

Proof. From (10), (26) and (28), we get

$$
\tilde{\operatorname{Ric}}(Y, Z)=\operatorname{Ric}(Y, Z)+\left(\frac{\tilde{r}-r}{2(n-1)^{2}}\right)[(2 n-3) g(Y, Z)-(n-2) \eta(Y) \eta(Z)]
$$

Which gives

$$
\begin{aligned}
& \tilde{\operatorname{Ric}}(Y, Z)-\left(\frac{\tilde{r}}{2(n-1)^{2}}\right)[(2 n-3) g(Y, Z)-(n-2) \eta(Y) \eta(Z)] \\
= & \operatorname{Ric}(Y, Z)-\left(\frac{r}{2(n-1)^{2}}\right)[(2 n-3) g(Y, Z)-(n-2) \eta(Y) \eta(Z)]
\end{aligned}
$$

Hence (33) shows the invariance of the tensor of $\eta$-Einstein.

\section{REFERENCES}

[1] Adati,T and Motsumoto,K.: On conformally recurrent and conformally symmetric P-Sasakian manifolds, TRU Maths., Vol.13(1977), 25-32.

[2] Adati, T. and Miyazawa, T.: Some Properties of P-Sasakian manifold. TRU. Maths. Vol.13(1), (1977), 33-42. 
[3] Biswas, S. C. and De, U. C. : Quarter symmetric metric connexion in an SPSasakian manifold. Commun. Fac. Sci. Univ. Ank. Series A1, Vol. 46 (1997), 49-56.

[4] Golab, S.: On semi-symmetric and quarter-symmetric linear connexions. Tensor, N. S., Vol. 29 (1975), 249-254.

[5] Mishra, R. S. and Pandey, S. N.: On quarter symmetric metric F-connexions. Tensor, N. S., Vol. (34), (1980), 1-7.

[6] Özgür, C.: On a class of Para-Sasakian manifolds. Turk. J. Math., Vol. (29), (2005), 249-257.

[7] Sato, I.: On structure similar to almost contact structure I. Tensor, N.S., Vol. 30 (1976), 219-224.

[8] Stavre, P.: On the S-concircular and S-conharmonic connections. Tensor, N. S., Vol. 38 (1982), 102-108.

[9] Tarafdar, D. and De, U. C.: On a type of P-Sasakian manifold. Extracta Mathematicae, Vol. 8 (1993), 31-36.

[10] Yano, K.: On semi-symmetric metric connection. Review of De Roumannie pure et applieques Math., (1970), 1554-1560.

Received: May, 2013 